\title{
BMJ Open Prevalence of diabetic retinopathy among 13473 patients with diabetes mellitus in China: a cross-sectional epidemiological survey in six provinces
}

\author{
Yan Liu, ${ }^{1}$ Yifan Song, ${ }^{1}$ Liyuan Tao, ${ }^{2}$ Weiqiang Qiu, ${ }^{1}$ Huibin Lv, ${ }^{1}$ Xiaodan Jiang, ${ }^{1}$ \\ Mingzhou Zhang, ${ }^{1}$ Xuemin $\mathrm{Li}^{1}$
}

To cite: Liu Y, Song Y, Tao L, et al. Prevalence of diabetic retinopathy among 13473 patients with diabetes mellitus in China: a crosssectional epidemiological survey in six provinces. BMJ Open 2017;7:e013199. doi:10.1136/bmjopen-2016013199

- Prepublication history for this paper is available online. To view these files please visit the journal online (http://dx.doi.org/10.1136/ bmjopen-2016-013199).

$\mathrm{YL}$ and $\mathrm{YS}$ contributed equally.

Received 4 July 2016 Revised 12 October 2016 Accepted 14 December 2016

\section{CrossMark}

\author{
${ }^{1}$ Department of \\ Ophthalmology, Peking \\ University Third Hospital, \\ Beijing, China \\ ${ }^{2}$ Research Center of Clinical \\ Epidemiology, Peking \\ University Third Hospital, \\ Beijing, China
}

Correspondence to Dr Xuemin Li;

Ixmlxm66@sina.com.cn

\section{ABSTRACT}

Objective: To describe the prevalence and severity of diabetic retinopathy (DR) and sight-threatening $D R$ (STDR) among Chinese adults with diabetes.

Design, setting and participants: A cross-sectional epidemiological survey across Mainland China $(\mathrm{N}=13$ 473). Participants were diagnosed with diabetes mellitus by physicians and transferred to our screening clinics (1/3 from hospital patients, $1 / 3$ from city residents, the other $1 / 3$ from rural residents). 2 fundus photographs were taken of each eye with a colourful, non-mydriatic and non-stereoscopic camera and were graded according to the UK guidelines.

Main outcomes and measures: The prevalence and severity of DR and STDR.

Results: Of the 13473 participants with diabetes participating in the study, 4591 had DR and 1769 had STDR, for an overall prevalence of $34.08 \%(95 \% \mathrm{Cl}$ $33.28 \%$ to $34.88 \%)$ and $13.13 \%(95 \% \mathrm{Cl} 12.56 \%$ to $13.70 \%$ ), respectively. Among these, gradable photographs were available for 12780 participants $(94.86 \%)$. Participants who were aged $>65$ years were less likely to suffer from DR or STDR $(p<0.001$; age distribution). No gender-related differences were present $(p>0.05)$. Participants with STDR suffered from more severe visual impairment compared with those without STDR $(p<0.001)$. The prevalence of DR and STDR in the northern region was higher than in the southern region $(p<0.001)$. The prevalence of $D R$ was closely related to duration of disease (OR 2.63; $95 \% \mathrm{Cl} 2.42$ to $2.86 ; p<0.001)$ and diabetes onset age (OR 0.38; $95 \% \mathrm{Cl} 0.35$ to $0.41 ; p<0.001$ ).

Conclusions: The prevalence of DR and STDR in Mainland China appeared a little high, and varied according to area. Non-proliferative DR was more common, but STDR needed prompt treatment, especially in economically less developed areas. This study highlights the necessity for DR screening and treatment in Mainland China.

\section{INTRODUCTION}

Diabetes mellitus (DM) is becoming a major threat to global public health. There were

\section{Strengths and limitations of this study}

- This is a cross-sectional epidemiological survey concerning diabetic retinopathy (DR) in a diabetic population (13 473 diabetic participants) across Mainland China (including both the northern and southern parts of China), which could comprehensively reflect $\mathrm{DR}$ prevalence status in the Chinese diabetic population.

- The participants included both hospital patients as well as rural and city residents.

- Despite the large sample size, the sampling method of this study was not stratified, resulting in a lack of representativeness.

- Owing to the large sample size and multiple screening centres, data collection was sometimes not comprehensive. Some invasive examination results such as blood glucose and glycated haemoglobin were incomplete, which resulted in a flaw in risk factor analysis.

382 million people who suffered from DM worldwide in 2013. The predicted number would be 592 million by 2035. Most people with diabetes live in low-income and middle-income countries and these will experience the greatest increase in cases of diabetes over the next 22 years. ${ }^{1}$ As the largest developing country in the world, China has experienced a rapid increase in the prevalence of DM and prediabetes in the past three decades. A recent diabetes epidemic meta-analysis indicated that the prevalence of diabetes has reached $9.7 \%$ in Mainland China, translating into 92.4 million adults with diabetes. ${ }^{2}$ Patients with diabetes are susceptible to diabetic retinopathy (DR). Since the prevalence of DM is increasing, so is the number of people with DR. DR is a common complication in individuals with $\mathrm{DM}$, which is caused by long-term damage to the small blood vessels in the retina. It has 
been established that DR can lead to severe, permanent visual impairment and is one of the most common causes of blindness in working-age adults (20-65 years). ${ }^{3}$ A previous report mentioned that after 15 years of diabetes, the prevalence of any DR would be $95 \%$ in insulin-dependent diabetes mellitus (IDDM) and 58\% in non-IDDM (NIDDM), while proliferative DR (PDR) would be $26 \%$ and $4 \%$ in IDDM and NIDDM, respectively. ${ }^{4}$

DR results in loss of quality of life for patients, as well as a significant burden to healthcare systems and society. Clinical trials have confirmed that good control of diabetes ${ }^{5}$ and other related risk factors (such as hypertension, exercise, smoking, etc) could significantly reduce the risk for DR. ${ }^{6-9}$ Proper treatment at the proper time during the progress of $\mathrm{DR}$ will reduce the risk of visual loss, as well as decrease the burden to individuals, healthcare systems and society. Focal/grid photocoagulation, panretinal photocoagulation and intravitreal injection of antivascular endothelial growth factor agents have been the standard of care to treat diabetic macular edema (DME) and DR, reducing the risk of vision loss in the long term. ${ }^{10-13}$ However, the awareness about DR among patients with diabetes is limited, and compliance with treatment is often poor. ${ }^{14}{ }^{15}$ China has experienced an economic blooming during the past 30 years, as well as the blood glucose level among Chinese citizens. ${ }^{16}$ Recent studies reported a rapid increasing prevalence of diabetes, especially in middle-aged high-risk men. ${ }^{17-18}$ What is worse, the treatment condition of DM is not satisfyingonly one-fourth of overall patients with diabetes were treated and only two-fifths of them had their glycated haemoglobin (HbA1c) controlled to a proper concentration $(<7.0 \%) .{ }^{19}$ Considering the poor management of hyperglycaemia, the prevalence and severity of DR may be higher than commonly expected. ${ }^{5}$

Although there are several researches that have studied the prevalence of DR in the Chinese population, ranging from $0.2 \%$ to $30.3 \%$ due to the different inclusion population, ${ }^{20}$ national population-based data on the prevalence and severity of DR in the diabetic population remain scarce. Most of the previous studies ${ }^{21-24}$ were implemented in one province or district, which are often economically developed regions. Also, most of these studies were conducted in the northern region of China. There are still few data for DR prevalence across Mainland China. Additionally, a majority of previous studies focused on regular residents, rather than the patients with diabetes, which resulted in a relatively low DR detection rate.

No recent comprehensive population-based estimate of the prevalence and severity of DR among participants with diabetes exists. This study is the first survey to investigate the prevalence and severity of DR in patients with diabetes in six provinces across Mainland China (including both the northern and southern parts of China).
METHODS

\section{Participants and screening procedure}

This study was a cross-sectional epidemiological survey, conducted from April 2014 to September 2015. Eight hospitals from six different provinces (Shandong, Guangxi, Guangdong, Henan, Inner Mongolia, Jilin Provinces) were included in this study. Participants were enrolled from both hospitals and local communities according to the study design (1/3 from hospital patients, $1 / 3$ from city residents and the other $1 / 3$ from rural residents). Hospital patients were diagnosed with DM and transferred to our screening clinics. Community participants were recruited by advertisements. They could visit the screening clinics and undergo screening examinations if they were previously diagnosed with DM by a qualified physician and consented to participate in this study. The necessity of screening was elaborated and screening procedures were provided for free for the participants. The research protocol was approved by the Ethics Committee of Peking University Third Hospital and written informed consent was obtained from all participants.

The screening procedure consisted of measuring visual acuity (VA) using the Early Treatment Diabetic Retinopathy Study (ETDRS) chart, checking the anterior segment structure (including the cornea, anterior chamber and lens) using slit lamp biomicroscopy of both eyes. Pupils would be dilated with $0.5 \%$ compound tropicamide eye drops (including $5 \mathrm{mg}$ tropicamide and $5 \mathrm{mg}$ phenylephrine hydrochloride per $1 \mathrm{~mL}$ ) if needed. Intraocular pressures were measured before and after pupil dilation. Digital, colourful and nonstereoscopic retinal photographs were taken by a nonmydriatic auto fundus camera (CR-1 Mark II, Canon, Tokyo, Japan), including two fields for each eye: one centred at the optic disc and the other at the macula. Patient information and digital images were randomly transmitted from screening clinics to one of the three grading centres (Peking University Third Hospital, Peking Union Medical College Hospital and Shantou International Eye Center) using the software named the VisDR Tele Diabetic Retinopathy Screening Platform (VisDR, V.1.1).

\section{Grading}

Grading was performed by trained and certified optometrists and ophthalmologists from three grading centres. Photographs were systematically graded using VisDR software, with a spatial resolution of $1024 * 768$ pixels. All of the graders underwent periodic tests to ensure the accuracy of grading. They examined fundus photographs for the presence and severity of DR according to the UK guidelines $^{25}$ (table 1). Each photograph underwent primary grading. All the photographs with any DR or other eye disease and $15 \%$ of the photographs without any DR were further graded by a secondary grader (another certified optometrist or ophthalmologist). If there was any different opinion in the grading of the 
Table1 DR classification used in our grading for DR screening based on the UK guidelines, Harding et a P $^{5}$

\begin{tabular}{|c|c|c|c|}
\hline \multicolumn{2}{|c|}{ Grade of retinopathy } & \multirow{2}{*}{$\begin{array}{l}\text { Description } \\
\text { None }\end{array}$} & \multirow{2}{*}{$\begin{array}{l}\text { Features } \\
\text { None }\end{array}$} \\
\hline Retinopathy & Ro & & \\
\hline & $\mathrm{R} 1$ & Mild NPDR & At least one microaneurysm \\
\hline & & & Retinal haemorrhages+any exudates \\
\hline & $\mathrm{R} 2$ & Severe NPDR & Venous beading \\
\hline & & & Venous loop or reduplication \\
\hline & & & IRMA \\
\hline & & & Multiple deep, round or blot haemorrhages \\
\hline & & & Cotton wool spots in addition to the above features \\
\hline & R3 & High-risk PDR & NVD \\
\hline & & & NVE \\
\hline & & & Pre-retinal or vitreous haemorrhage \\
\hline & & & Extensive fibrovascular proliferation \\
\hline & & & Pre-retinal fibrosis \pm tractional retinal detachment \\
\hline \multirow[t]{5}{*}{ Maculopathy } & MO & & No maculopathy \\
\hline & M1 & & Exudate within one DD of entry of fovea \\
\hline & & & Circinate or group of exudates within the macula \\
\hline & & & $\begin{array}{l}\text { Retinal thickening less than or equal to one DD of centre of fovea (if stereo } \\
\text { available) }\end{array}$ \\
\hline & & & $\begin{array}{l}\text { Any microaneurysm or haemorrhage within one DD of centre of fovea only } \\
\text { if VA (if no stereo) } \leq 6 / 12\end{array}$ \\
\hline Photocoagulation & $\mathrm{P}$ & & Focal or scatter laser scars \\
\hline Other lesions & OL & & Other lesions such as non-diabetic lesions as AMD \\
\hline Unclassifiable & $\mathrm{U}$ & Ungradable & For example, if media opacity, poor view on photographs \\
\hline
\end{tabular}

same assigned photograph, it would be assessed by a third grader (an authoritative ophthalmologist) who would make the final decision. Therefore, the final decision could be made by the primary grader if negative, by agreement between the primary and secondary grader or by an authoritative grader if any DR or other eye disease was present. Sight-threatening DR (STDR) was identified as present if any features of maculopathy (M1), pre-PDR (R2) or PDR (R3) were found. Photographs that were not assessable or could not be graded were considered ungradable (U).

The DR grading for each participant was based on the worse DR grades of the two eyes. If one eye was non-STDR (R0 or R1) and the other eye was graded as $\mathrm{U}$, the participant would be classified as $\mathrm{U}$ and transferred to the ophthalmology clinic for further examination. If one eye was STDR (R2, R3 or M1) and the other eye was $\mathrm{U}$, the participant would be classified as STDR and transferred. If non-STDR (R0 or R1) was graded in both eyes, participants would be graded as non-STDR and not transferred. The better VA of the two eyes represented the participant's VA. It was also noted by the graders if there were any other eye diseases presented, which included but were not limited to increased cup-or-disc ratio, cataract, macular degeneration, retinal artery occlusion, retinal vein occlusion, anterior ischaemic optic neuropathy, macular hole, epiretinal membrane and hypermyopia fundus changes. These participants were also transferred to a specialist clinic.

\section{Data collection}

VisDR software stored all the screening data, including demographic information, VA, screening hospitals and fundus pictures. The ETDRS VA was converted into Snellen VA and uploaded in the DR grading software. VA was classified into three groups: no visual impairment or mild visual impairment (no-VI, VA $\geq 20 / 66$ ), moderate visual impairment (mod-VI, 20/66>VA $\geq 20 / 200)$ and severe visual impairment $(\mathrm{s}-\mathrm{VI}, \mathrm{VA}<20 / 200) .{ }^{26}$ At the time of the clinical visit, the following measurements were recorded, including body mass index (BMI, calculated from measured height and weight), diabetes duration and diabetes types.

\section{Statistical analysis}

Statistical analyses were performed using SPSS V.18.0 (SPSS, Chicago, Illinois, USA) software. Descriptive analyses included the prevalence of DR and STDR in the overall diabetic population, and were calculated by age group ( $\leq 65$ and $>65)$, gender and region with $95 \%$ CIs. Student's t-test was used to compare continuous variables, and the $\chi^{2}$ test was used to compare proportions among groups. The level of visual impairment was recorded and evaluated, which was compared between the groups with and without STDR, or in different regions using the $\chi^{2}$ test. The duration of diabetes was calculated as the difference between the year of diagnosis (as reported by the participant) and the year of this study. Those with newly diagnosed diabetes were given a diabetes duration of 0 years. All participants classified as 
$\mathrm{U}$ were excluded in this step. An $\alpha$ level of 0.05 was adopted as the significance level.

\section{RESULTS}

A screening procedure was performed at eight hospitals in eight cities from six different provinces in China from April 2014 to October 2015. A total of 13473 participants were included in this study, including $0.71 \%$ with type 1 diabetes (96/13 473), 98.75\% with type 2 diabetes $(13304 / 13473)$ and $0.54 \%$ with pregnancy diabetes (73/13 473) with a median duration of 6.0 (p25: 3.0, p75: 11.0) years. The average age of all participants included was $62.46 \pm 10.45$ while the average BMI (response rate $66.0 \%, 8890 / 13473$ ) was $24.76 \pm 3.70$. About half of the participants were female (7310/ 13473 ; 54.30\%). Among all the fundus photographs, $5.14 \%(693 / 13473)$ were graded as ungradable for one or both eyes (mostly because of poor image quality due to ocular media opacity or a too small pupil), which is in accordance with the UK national minimum standard of $<10 \% .^{27}$

The estimated prevalence of any DR among all subjects with diabetes was $34.08 \%(4591 / 13473,95 \%$ CI $33.28 \%$ to $34.88 \%$ ) and of any STDR was $13.13 \%$ (1769/ $13473,95 \%$ CI $12.56 \%$ to $13.70 \%)$. The most common DR status was R1M0, with a prevalence of $15.80 \%$. The estimated prevalence of each status is demonstrated in table 2. The prevalence of any DR and STDR in those with type 1 diabetes was $31.5 \%$ and $18.5 \%$, in type 2 diabetes was $30.5 \%$ and $13.8 \%$, and in pregnancy diabetes was $29.9 \%$ and $9.0 \%$, respectively. No significant difference was found in gender of both DR $(31.13 \%$ vs $29.97 \%, \mathrm{p}=0.13)$ and STDR ( $13.88 \%$ vs $13.81 \%, \mathrm{p}=0.98)$ prevalence as demonstrated in figure 1 . We also found that older people ( $>65$ years old) were less likely to suffer from DR or STDR (25.65\% vs $33.77 \%, \mathrm{p}<0.001$; $11.07 \%$ vs $15.70 \%, \mathrm{p}<0.001)$.

There were 30 participants out of 13473 (missing rate $0.22 \%$ ) who did not report VA. Within the remaining

Table 2 Estimated prevalence of DR and STDR in individuals with diabetes $(\mathrm{N}=13473)$

\begin{tabular}{lrll}
\hline DR grading & N & Percent & 95\% Cl \\
\hline Non-STDR & 11011 & 81.73 & 81.06 to 82.37 \\
R0M0 & 8882 & 65.92 & 65.12 to 66.72 \\
R1M0 & 2129 & 15.80 & 15.19 to 16.43 \\
STDR & 1769 & 13.13 & 12.57 to 13.71 \\
R2M0 & 95 & 0.71 & 0.57 to 0.87 \\
R3M0 & 120 & 0.89 & 0.74 to 1.07 \\
R1M1 & 940 & 6.98 & 6.56 to 7.42 \\
R2M1 & 306 & 2.27 & 2.03 to 2.54 \\
R3M1 & 308 & 2.29 & 2.04 to 2.56 \\
Ungradable & 693 & 5.14 & 4.78 to 5.53 \\
\hline
\end{tabular}

DR, diabetic retinopathy; M0, no maculopathy; M1, maculopathy; R0, no diabetic retinopathy; R1, background diabetic retinopathy; $\mathrm{R} 2$, preproliferative diabetic retinopathy; $\mathrm{R} 3$, proliferative diabetic retinopathy; STDR, sight-threatening diabetic retinopathy.

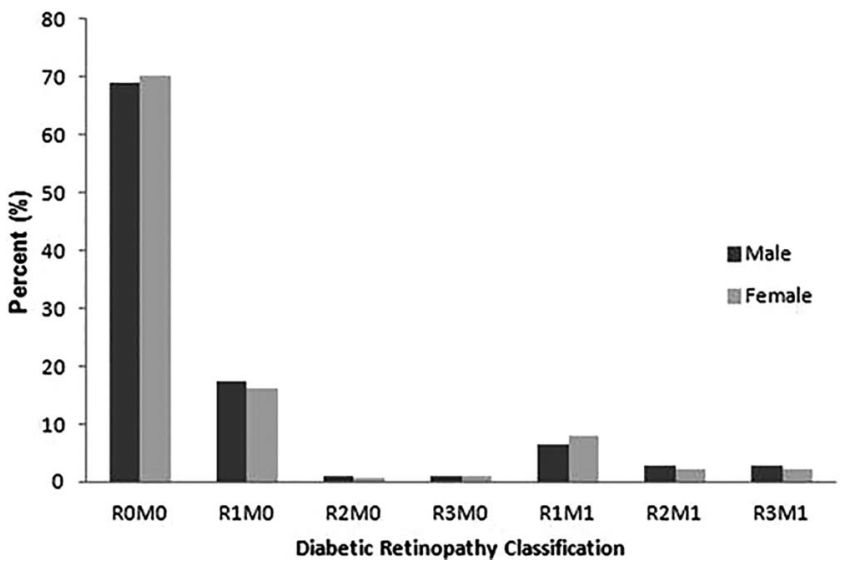

Figure 1 Prevalence of diabetic retinopathy classification by gender. Diabetic retinopathy was classified according to UK guidelines as demonstrated in table 1. There was no statistically significant difference between male and female subjects $\left(\chi^{2}\right.$ test, $\left.\mathrm{p}>0.05\right)$.

13443 participants, $1.65 \%$ (222/13 443), $7.56 \%$ (1016/ $13443)$ and $90.79 \%(12$ 205/13 443) participants were grouped as those with s-VI, mod-VI and no-VI, respectively. After excluding 693 participants with ungradable photographs, participants with STDR had a significantly higher risk of visual impairment than those without STDR $(\mathrm{p}<0.001)$. Detailed data were demonstrated in table 3 .

China can be divided into the northern and southern parts by the Qinling Mountains-Huaihe River Line. Table 4 showed the estimated prevalence of DR and STDR in the southern and northern regions of China. In total, $46.63 \%$ of the participants lived in the south, while $53.37 \%$ lived in the north. A significant difference was detected between the southern and northern populations in regard to the prevalence of any DR $(27.55 \%$ vs $37.07 \%, \mathrm{p}<0.001)$. Living in northern China was a risk factor for DR (OR 1.30; 95\% CI 1.20 to 1.40). Also, the prevalence of STDR was higher in the northern region than in the southern region $(11.91 \%$ vs $15.53 \%$, OR 1.36 ; $95 \%$ CI 1.23 to $1.51 ; \mathrm{p}<0.001)$. Interestingly, on the other hand, the southern population experienced a more s-VI $(\mathrm{p}<0.001)$ as shown in table 5. Additionally, longer diabetes duration ( $>10$ years) was associated with a higher prevalence of DR and STDR (OR 2.63; 95\% CI 2.42 to $2.86 ; \mathrm{p}<0.001$; OR $2.73 ; 95 \%$ CI 2.46 to 3.03 ; $\mathrm{p}<0.001)$. Also, we found that participants with older diabetes onset age ( $>50$ years old) were less likely to develop DR (OR $0.38 ; 95 \%$ CI 0.35 to $0.41 ; \mathrm{p}<0.001$ ) or STDR (OR 0.35; $95 \%$ CI 0.31 to $0.38 ; \mathrm{p}<0.001$ ).

\section{DISCUSSION}

The prevalence of DR and STDR in this study was $34.08 \%$ and $13.13 \%$, respectively. DR prevalence has been reported in many other researches, but comparisons are difficult to make due to various participant inclusion criteria and different DR definitions, since 
Table 3 Visual impairment levels in general, and by groups with and without STDR

\begin{tabular}{|c|c|c|c|c|c|c|c|c|}
\hline & \multicolumn{2}{|l|}{ Total } & \multirow[b]{2}{*}{$\mathbf{N}$} & \multicolumn{2}{|c|}{ Non-STDR } & \multicolumn{2}{|l|}{ STDR } & \multirow[b]{2}{*}{ p Value } \\
\hline & $\mathbf{N}$ & Per cent & & $\mathbf{N}$ & Per cent & $\mathbf{N}$ & Per cent & \\
\hline S-VI & 222 & 1.65 & 154 & 85 & 0.77 & 69 & 3.92 & $<0.001$ \\
\hline mod-VI & 1016 & 7.56 & 897 & 616 & 5.60 & 281 & 15.97 & \\
\hline no-VI & 12205 & 90.79 & 11716 & 10306 & 93.63 & 1410 & 80.11 & \\
\hline
\end{tabular}

our programme was conducted according to the UK guidelines, the results of which could be compared with those who followed the same guidelines. The prevalence of DR and STDR was $25.3 \%$ and $6.0 \%$ in a systematic screening in Liverpool, ${ }^{28}$ and was $30.9 \%$ and $2.9 \%$ in a national screening in Wales among patients with type 2 diabetes. ${ }^{29}$ Both results are lower than the findings of our study ( $34.1 \%$ and $13.1 \%$, respectively). One possible reason might be the shorter DM duration of the participants included in those two studies when compared with ours-the median duration of DM was 3.2 years in the Liverpool study and 5.3 years in the Wales study, while it was 6.0 years in our study. In Hong Kong, a higher prevalence of $39.0 \%$ DR was reported, as well as an accordingly longer mean duration of 7.7 years. ${ }^{30}$ However, the prevalence of STDR in this study was higher than that in Hong Kong $(13.13 \%$ vs $9.8 \%$ ), which might be due to a lack of prompt and effective treatment. It can be concluded that DR has occurred in about one-third of the diabetes population in our study, and over $10 \%$ of these individuals have visual impairment. Among those who have STDR, $19.89 \%$ have visual impairment, and the appearance of mod-VI and s-VI was $15.97 \%$ and $3.92 \%$, respectively, higher than those in Hong Kong $(9.8 \%, 7.7 \%$ and $2.1 \%$, respectively). It has been mentioned that in China, 7 out of 10 patients with diabetes are not aware of their high blood glucose condition, ${ }^{19}$ which means participants may have a much longer history than reported. This may be one possible reason for the higher percentage of STDR and visual impairment. Therefore, it should be strongly suggested that a fundus screening should be taken as soon as the patients are diagnosed with diabetes. What is worse, it was reported among the patients with diabetes that only one-fourth of the overall patients with diabetes were treated. And among treated patients less than a $1 / 2$ patients' blood sugar can reach the goal level and $2 / 5$ of patients had their HbAlc controlled to a proper concentration. ${ }^{19}$ Considering that both the HbAlc level and variability are closely associated with the occurrence and severity of $\mathrm{DR},{ }^{5}$ it might be one important reason why STDR prevalence in this study is higher than that in the UK and Hong Kong. A meta-analysis of population-based studies in Mainland China reported the prevalence of any DR as $23 \%(95 \%$ CI $17.8 \%$ to $29.2 \%)$, non-PDR as $19.1 \%(13.6 \%$ to $26.3 \%)$ and PDR as $2.8 \%(1.9 \%$ to $4.2 \%$ ) among those with DM, but the included studies varied by examination methods and DR definitions, while the prevalence of STDR and maculopathy was not reported. ${ }^{20}$ The comparison of this study to related studies is demonstrated in table 5 .

According to our findings, about one-third of patients with DM in Mainland China suffered from DR, and among them more than one-third need further medical assessment and treatment. We found that visual impairment was more severe in the patients with STDR than the non-STDR population, which should arouse more attention. Although how much of the visual impairment can be attributed to STDR is not clear, we did find that STDR was associated with visual impairment. Good management of blood glucose and prompt treatment of retinopathy (including maculopathy) is the key to avoid permanent visual impairment. In this study, we did not determine the cause of the visual impairment or whether it was correctable, but all patients with STDR or

Table 4 Estimated prevalence of DR and STDR, BMI and visual impairment in southern and northern regions

\begin{tabular}{lllllrr}
\hline & Type & Southern region & Per cent & Northern region & Per cent & p Value \\
\hline DR & Non-DR & 4317 & 72.45 & 4565 & 66.93 & $<0.001$ \\
& DR & 1642 & 27.55 & 2256 & 33.07 & $<4.47$ \\
STDR & Non-STDR & 5249 & 88.09 & 5762 & 15.53 & $<0.001$ \\
& STDR & 710 & 11.91 & 1059 & $<0.001$ \\
BMI & & $24.21 \pm 3.54$ & & $25.33 \pm 3.77$ & 1.24 \\
VI & S-VI & 132 & 2.14 & 90 & 7.95 & $<0.001$ \\
& mod-VI & 438 & 7.10 & 578 & 90.81 \\
\hline
\end{tabular}

BMI, body mass index; DR, diabetic retinopathy; mod-VI means moderate visual impairment; no-VI means no or mild visual impairment; STDR, sight-threatening diabetic retinopathy; s-VI means severe visual impairment; VI, visual impairment. 
Table 5 Comparison of this study to similar studies using the UK guidelines

\begin{tabular}{lclll}
\hline District & Sample size & Screening time & Prevalence of DR (\%) & Prevalence of STDR (\%) \\
\hline Liverpool & 8062 & $1991-1999$ & 45.7 (type 1) & 16.4 (type 1) \\
Wales & & & 25.3 (type 2) & 6.0 (type 2) \\
& 91393 & $2005-2009$ & 56.0 (type 1) & 11.2 (type 1) \\
Hong Kong & 174532 & $2010-2014$ & 30.3 (type 2) & 9.8 \\
Mainland China & 13473 & $2014-2015$ & 34.0 & 13.1 \\
\hline
\end{tabular}

Type 1 means type 1 diabetes; type 2 means type 2 diabetes.

DR, diabetic retinopathy; STDR, sight-threatening diabetic retinopathy.

other eye diseases who required treatment were referred to ophthalmologists for further assessment.

Mainland China can be divided into the northern and southern parts by the Qinling Mountains-Huaihe River Line. Our investigation was conducted in eight different cities in six provinces, among which five cities are located in the northern region while the other three are in the southern region. According to our research, the prevalence of DR and STDR was higher in the northern region (33.07\% and $15.53 \%)$ compared with the southern region $(27.55 \%$ and $11.91 \%$ ), consistent with previous studies. ${ }^{20}$ The discrepancy of DR prevalence between the north and the south was most likely due to the differences of lifestyle and socioeconomic status. For example, gross domestic product per capita levels in the north is higher than that in the south, so as the BMI levels and prevalence of overweight. ${ }^{31}$ Since the occurrence of diabetes can be largely influenced by socioeconomic and lifestyle change, social urbanisation, accompanied by drastic lifestyle changes, such as physical inactivity, unhealthy diet, obesity and hypertension caused by stress, may cause the rise of incidence both in DM and DR. Previous studies also showed that BMI and the prevalence of central obesity was higher in the residents of northern compared with southern in China, ${ }^{32}$ and this may also contribute to the higher DR rate in the northern region. Conversely, despite the higher prevalence of DR in the north, visual impairment was more severe in the south, probably due to the economically less developed status and poor management of blood glucose levels.

In this study, longer duration of diabetes and earlier onset age were identified as risk factors for DR and STDR. Duration of diabetes has long been considered to be related to the prevalence of DR. Similar results were also reported by Liu $e t a l^{33}$ and Wang $e t a l^{34}$ in a Chinese sample of people. In a non-Chinese population, the Wisconsin Epidemiologic Study of Diabetic Retinopathy (WESDR) had revealed that the prevalence of any retinopathy in patients with type 1 diabetes was $8 \%$ at a duration of 3 years diabetes, $25 \%$ at a duration of 5 years diabetes, $60 \%$ at a duration of 10 years diabetes and $80 \%$ at a duration of 15 years diabetes. The prevalence of PDR was $0 \%$ at a duration of 3 years diabetes and increased to $25 \%$ at a duration of 15 years diabetes. ${ }^{35}$ Moreover, our finding of the relationship between diabetes onset age and DR prevalence was also reported previously. Raman $e t a l^{36}$ reported that the prevalence of DR was almost twice more in those participants who developed diabetes before the age of 40 years than in those who developed it later. The results suggest that an annual retinal examination and early detection of DR could considerably reduce the risk of visual loss in individuals with diabetes, especially in early onset diabetes, and its importance increases with the elongation of diabetes duration.

Our current study has several limitations. Although our participants included a large sample of the diabetic population, both from hospital patients and community individuals, our sampling method was not stratified, resulting in a lack of representativeness. To get a better view of the present prevalence of DR in China, we included eight cities in six different provinces, which contains five northern cities (in four different provinces) and three southern cities (in two different provinces). However, it is still just part of China. To have more specific consideration, a nationwide, population-based research needs to be taken in the future. Another major problem came down to the low response of blood glucose and HbA1c levels of participants in our investigation $(<30 \%)$. As a result, we did not include or analyse this part of results in this study considering the nonrespondent bias, which made the analysis of the relationship between blood glucose control and DR prevalence impossible. In addition, the VA we recorded in this study was the presenting VA, not best corrected VA, which might be a little inaccurate when estimating the extent of visual impairment causing by DR.

\section{CONCLUSION}

In summary, we report a high prevalence of DR and STDR in six provinces across Mainland China, and the prevalence of DR and STDR varied according to areas. This study highlights the necessity for comprehensive screening and timely treatment of DR in China.

Contributors $X L$ had full access to all of the data in the study and takes responsibility for the integrity of the data. $X L, Y L, Y S$ and $W Q$ were involved in study concept and design. YL, YS, HL, XJ and MZ were involved in acquisition of data. $L T, Y L$ and $Y S$ were involved in analysis and interpretation of data. $Y L$ and $Y S$ were involved in drafting of the manuscript. $X L, W Q$ and 
$\mathrm{HL}$ were involved in critical revision of the manuscript. $\mathrm{LT}$ and $\mathrm{YL}$ were involved in statistical analysis. $\mathrm{XL}$ and $\mathrm{WQ}$ were involved in study supervision.

Funding This study was supported by the Beijing Municipal Science and Technology Commission. Grant number: Z141107002514042.

Competing interests None declared.

Patient consent Obtained.

Provenance and peer review Not commissioned; externally peer reviewed.

Data sharing statement No additional data are available.

Open Access This is an Open Access article distributed in accordance with the Creative Commons Attribution Non Commercial (CC BY-NC 4.0) license, which permits others to distribute, remix, adapt, build upon this work noncommercially, and license their derivative works on different terms, provided the original work is properly cited and the use is non-commercial. See: http:// creativecommons.org/licenses/by-nc/4.0/

\section{REFERENCES}

1. Guariguata L, Whiting DR, Hambleton I, et al. Global estimates of diabetes prevalence for 2013 and projections for 2035. Diabetes Res Clin Pract 2014;103:137-49.

2. Zuo H, Shi Z, Hussain A. Prevalence, trends and risk factors for the diabetes epidemic in China: a systematic review and meta-analysis. Diabetes Res Clin Pract 2014;104:63-72.

3. Zheng $\mathrm{Y}, \mathrm{He} \mathrm{M}$, Congdon $\mathrm{N}$. The worldwide epidemic of diabetic retinopathy. Indian J Ophthalmol 2012;60:428-31.

4. Viswanath K, McGavin DD. Diabetic retinopathy: clinical findings and management. Community Eye Health 2003;16:21-4.

5. Hermann JM, Hammes HP, Rami-Merhar B, et al. HbA1c variability as an independent risk factor for diabetic retinopathy in type 1 diabetes: a German/Austrian multicenter analysis on 35,891 patients. PLOS ONE 2014;9:e91137.

6. Kawasaki R, Tanaka S, Tanaka S, et al. Incidence and progression of diabetic retinopathy in Japanese adults with type 2 diabetes: 8 year follow-up study of the Japan Diabetes Complications Study (JDCS). Diabetologia 2011:54:2288-94.

7. Pugliese G, Solini A, Zoppini G, et al. High prevalence of advanced retinopathy in patients with type 2 diabetes from the Renal Insufficiency And Cardiovascular Events (RIACE) Italian Multicenter Study. Diabetes Res Clin Pract 2012;98:329-37.

8. Boule NG, Kenny GP, Haddad E, et al. Meta-analysis of the effect of structured exercise training on cardiorespiratory fitness in type 2 diabetes mellitus. Diabetologia 2003;46:1071-81.

9. Hammes HP, Kerner W, Hofer S, et al. Diabetic retinopathy in type 1 diabetes-a contemporary analysis of 8,784 patients. Diabetologia 2011;54:1977-84.

10. Abu El-Asrar AM. Evolving strategies in the management of diabetic retinopathy. Middle East Afr J Ophthalmol 2013;20:273-82.

11. Do DV, Nguyen QD, Boyer D, et al. One-year outcomes of the da Vinci Study of VEGF Trap-Eye in eyes with diabetic macular edema. Ophthalmology 2012;119:1658-65.

12. Mitchell $P$, Bandello $F$, Schmidt-Erfurth $U$, et al. The RESTORE study: ranibizumab monotherapy or combined with laser versus laser monotherapy for diabetic macular edema. Ophthalmology 2011;118:615-25.

13. Solaiman KA, Diab MM, Dabour SA. Repeated intravitreal bevacizumab injection with and without macular grid photocoagulation for treatment of diffuse diabetic macular edema. Retina 2013;33:1623-9.

14. Zhou M, Astell-Burt T, Bi Y, et al. Geographical variation in diabetes prevalence and detection in China: multilevel spatial analysis of 98,058 adults. Diabetes Care 2015;38:72-81.
15. Munoz B, O'Leary M, Fonseca-Becker F, et al. Knowledge of diabetic eye disease and vision care guidelines among Hispanic individuals in Baltimore with and without diabetes. Arch Ophthalmol 2008;126:968-74.

16. World HO. Raised fasting blood glucose $(\geq 7.0 \mathrm{mmol} / \mathrm{L}$ or on medication): data by country. Vol 2015. 2015.

17. Yang L, Shao J, Bian Y, et al. Prevalence of type 2 diabetes mellitus among inland residents in China (2000-2014): a meta-analysis. $J$ Diabetes Investig 2016;7:845-52.

18. Wang $\mathrm{C}$, Zhang $\mathrm{Y}$, Zhang $\mathrm{L}$, et al. Prevalence of type 2 diabetes among high-risk adults in Shanghai from 2002 to 2012. PLoS ONE 2014;9:e102926.

19. $\mathrm{Xu} \mathrm{Y}$, Wang $\mathrm{L}, \mathrm{He} \mathrm{J}$, et al. Prevalence and control of diabetes in Chinese adults. JAMA 2013;310:948-59.

20. Liu L, Wu X, Liu L, et al. Prevalence of diabetic retinopathy in mainland China: a meta-analysis. PLoS ONE 2012;7:e45264.

21. Xie $X, X u L$, Yang $H$, et al. Frequency of diabetic retinopathy in the adult population in China: the Beijing Eye Study 2001. Int Ophthalmol 2009;29:485-93.

22. Wang $F H$, Liang $Y B$, Peng $X Y$, et al. Risk factors for diabetic retinopathy in a rural Chinese population with type 2 diabetes: the Handan Eye Study. Acta Ophthalmol 2011;89:e336-43.

23. Pang $\mathrm{C}$, Jia $\mathrm{L}$, Jiang $\mathrm{S}$, et al. Determination of diabetic retinopathy prevalence and associated risk factors in Chinese diabetic and pre-diabetic subjects: Shanghai diabetic complications study. Diabetes Metab Res Rev 2012;28:276-83.

24. Xu J, Wei WB, Yuan MX, et al. Prevalence and risk factors for diabetic retinopathy: the Beijing Communities Diabetes Study 6. Retina 2012:32:322-9.

25. Harding S, Greenwood R, Aldington S, et al. Grading and disease management in national screening for diabetic retinopathy in England and Wales. Diabet Med 2003;20:965-71.

26. Roy FH. World blindness: definition, incidence and major treatable causes. Ann Ophthalmol 1974:6:1049-50.

27. Committee UNS. Essential elements in developing a diabetic retinopathy screening programme. Workbook version 4.4 ed. Committee UNS, 2012

28. Younis N, Broadbent DM, Harding SP, et al. Prevalence of diabetic eye disease in patients entering a systematic primary care-based eye screening programme. Diabet Med 2002;19:1014-21.

29. Thomas RL, Dunstan FD, Luzio SD, et al. Prevalence of diabetic retinopathy within a national diabetic retinopathy screening service. Br J Ophthalmol 2015;99:64-8.

30. Lian JX, Gangwani RA, McGhee SM, et al. Systematic screening for diabetic retinopathy (DR) in Hong Kong: prevalence of DR and visual impairment among diabetic population. Br J Ophthalmol 2016;100:151-5.

31. Zhuo Q, Wang Z, Piao J, et al. Geographic variation in the prevalence of overweight and economic status in Chinese adults. Br J Nutr 2009;102:413-18.

32. Reynolds K, Gu D, Whelton PK, et al. Prevalence and risk factors of overweight and obesity in China. Obesity (Silver Spring) 2007:15:10-18.

33. Liu L, Wu J, Yue S, et al. Incidence density and risk factors of diabetic retinopathy within type 2 diabetes: a five-year cohort study in China (report 1). Int J Environ Res Public Health 2015;12:7899-909.

34. Wang FH, Liang YB, Zhang F, et al. Prevalence of diabetic retinopathy in rural China: the Handan Eye Study. Ophthalmology 2009;116:461-7.

35. Klein R, Knudtson MD, Lee KE, et al. The Wisconsin Epidemiologic Study of Diabetic Retinopathy: XXII the twenty-five-year progression of retinopathy in persons with type 1 diabetes. Ophthalmology 2008;115:1859-68.

36. Raman R, Vaitheeswaran K, Vinita K, et al. Is prevalence of retinopathy related to the age of onset of diabetes? Sankara Nethralaya Diabetic Retinopathy Epidemiology and Molecular Genetic Report No. 5. Ophthalmic Res 2011;45:36-41. 